\title{
Scattering and bound states of spinless particles in a mixed vector-scalar smooth step potential
}

\author{
M.G. Garcia, A.S. de Castro ${ }^{1}$ \\ UNESP - Campus de Guaratinguetá \\ Departamento de Física e Química \\ 12516-410 Guaratinguetá SP - Brazil
}

${ }^{1}$ E-mail address: castro@pq.cnpq.br 


\begin{abstract}
Scattering and bound states for a spinless particle in the background of a kink-like smooth step potential, added with a scalar uniform background, are considered with a general mixing of vector and scalar Lorentz structures. The problem is mapped into the Schrödinger-like equation with an effective Rosen-Morse potential. It is shown that the scalar uniform background present subtle and trick effects for the scattering states and reveals itself a high-handed element for formation of bound states. In that process, it is shown that the problem of solving a differential equation for the eigenenergies is transmuted into the simpler and more efficient problem of solving an irrational algebraic equation.
\end{abstract}




\section{Introduction}

The one-dimensional step potential is of certain interest to model the transition between two structures. In solid state physics, for example, a step-like potential which changes continuously over an interval whose dimensions are of the order of the interatomic distances can be used to model the average potential which holds the conduction electrons in metals. In the presence of strong potentials, though, the Schrödinger equation must be replaced by their relativistic counterparts. The scattering of spin- $1 / 2$ particles by a square step potential, considered as a time component of a vector potential, is well-known and crystalized in textbooks [1]. In that scenario it appears the celebrated Klein's paradox [3]. The analysis of the same problem with the Klein-Gordon (KG) equation was not neglected [2, 4]. The background of the kink configuration of the $\phi^{4}$ model $(\tanh \gamma x)$ [5] is of interest in quantum field theory where topological classical backgrounds are responsible for inducing a fractional fermion number on the vacuum. Models of these kinds, known as kink models are obtained in quantum field theory as the continuum limit of linear polymer models [6]. In a recent paper the complete set of bound states of fermions in the presence of this sort of kink-like smooth step potential has been addressed by considering a pseudoscalar coupling in the Dirac equation [7]. A peculiar feature of the kink-like potential is the absence of bounded solutions in a nonrelativistic theory because it gives rise to an ubiquitous repulsive potential. Of course this problem neatly reveals that our nonrelativistic preconceptions are mistaken.

It is well known from the quarkonium phenomenology that the best fit for meson spectroscopy is found for a convenient mixture of vector and scalar potentials put by hand in the equations (see, e.g. [12]). The same can be said about the treatment of the nuclear phenomena describing the influence of the nuclear medium on the nucleons [13]. It happens that when the vector and scalar potentials fulfill the conditions for spin and pseudospin symmetries, i.e. they have the same magnitude, the energy spectrum does not depend on the spinorial structure, being identical to the spectrum of a spinless particle [14.

In the present work the scattering a spinless particle in the background of a kink-like smooth step potential, added with a scalar uniform background, is considered with a general mixing of vector and scalar Lorentz structures. Although the scalar potential finds many of their applications in nuclear and particle physics, it could also simulate an effective mass term in solid state 
physics and so it could be useful for modelling transitions between structures such a Josephson junctions [15]. It is often useful, because of simplicity, to approximate the behavior of relativistic fermions by spinless particles obeying the $\mathrm{KG}$ equation. It turns out that some results almost do not depend on the spin structure of the particle, e.g. the onset of scaling in some structure functions in the case of relativistic quark models used for studying quarkhadron duality [8], the photoelectron spectra in the strong field laser-induced ionization and recollision process [9], the electric polarizability of the ground state of a particle bound in a strong Coulomb field [10], and the differential scattering cross section for forward scattering [11]. Nevertheless, our purpose is to investigate the basic nature of the phenomena without entering into the details involving specific applications. In other words, the aim of this paper is to search new solutions of a fundamental equation in physics which can be of help to see more clearly what is going on into the details of a more specialized and complex circumstance. In passing, it is shown that a serious problem with the square step potential, overlooked in the literature, does not manifest for the smooth step potential. Our problem is mapped into an exactly solvable Sturm-Liouville problem of a Schrödinger-like equation with an effective Rosen-Morse potential which been applied in discussing polyatomic molecular vibrational states [16]. The scalar uniform background makes its influence not only for the scattering states but reveals itself a high-handed element for formation of bound states. In that process, the problem of solving a differential equation for the eigenenergies is transmuted into the simpler and more efficient problem of solving an irrational algebraic

equation. With this methodology the whole relativistic spectrum is found, if the particle is massless or not. Nevertheless, bounded solutions do exist only under strict conditions.

\section{The KG equation with vector and scalar potentials}

The (1+1)-dimensional KG equation for a free particle of rest mass $m$ corresponding to the relativistic energy-momentum relation $E^{2}=c^{2} p^{2}+m^{2} c^{4}$, where the energy $E$ and the momentum $p$ are substituted by operators, $i \hbar \partial / \partial t$ and $-i \hbar \partial / \partial x$ respectively, acting on the wave function $\Phi(x, t)$. Here, $c$ is the speed of light and $\hbar$ is the Planck constant $(\hbar=h /(2 \pi))$. In the pres- 
ence of external potentials the energy-momentum relation becomes

$$
\left(E-V_{t}\right)^{2}=c^{2}\left(p-\frac{V_{s p}}{c}\right)^{2}+\left(m c^{2}+V_{s}\right)^{2}
$$

where the subscripts for the potentials denote their properties under a Lorentz transformation: $t$ and $s p$ for the time and space components of a vector potential, and $s$ for the scalar potential. A continuity equation for the $\mathrm{KG}$ equation

$$
\frac{\partial \rho}{\partial t}+\frac{\partial J}{\partial x}=0
$$

is satisfied with $\rho$ and $J$ defined as

$$
\begin{aligned}
\rho & =\frac{i \hbar}{2 m c^{2}}\left(\Phi^{*} \frac{\partial \Phi}{\partial t}-\frac{\partial \Phi^{*}}{\partial t} \Phi\right)-\frac{V_{t}}{m c^{2}}|\Phi|^{2} \\
J & =\frac{\hbar}{2 i m}\left(\Phi^{*} \frac{\partial \Phi}{\partial x}-\frac{\partial \Phi^{*}}{\partial x} \Phi\right)-\frac{V_{s p}}{m c}|\Phi|^{2}
\end{aligned}
$$

Note that the KG equation is covariant under the charge-conjugation operation, meaning that the KG equation remains invariant under the simultaneous transformations $\Phi \rightarrow \pm \Phi^{*}, E \rightarrow-E, p \rightarrow-p, V_{s p} \rightarrow-V_{s p}$ and $V_{t} \rightarrow-V_{t}$. In other words, if $\Phi$ is a solution for a particle (antiparticle) with energy $E$ and momentum $p$ for the potentials $V_{t}, V_{s p}$ and $V_{s}$, then $\pm \Phi^{*}$ is a solution for a antiparticle (particle) with energy $-E$ and momentum $-p$ for the potentials $-V_{t},-V_{s p}$ and $V_{s}$. Note also that $\rho \rightarrow-\rho$ and $J \rightarrow-J$. These last results are of particular importance to interpret $\rho$ and $J$ as charge density and charge current density, respectively, and to recognize that the vector potential couples with the charge of the particle/antiparticle whereas the scalar potential couples with the mass, as one could suspect from the appearance of $V_{s}$ in (11) and from the absence of $V_{s}$ in (3). Furthermore, the change $E \rightarrow-E$ and related change $i \hbar \partial / \partial t \rightarrow-i \hbar \partial / \partial t$ permit us to conclude that if the particle travels forward in time then the antiparticle travels backward in time.

For time-independent potentials the KG equation admits solutions in the form

$$
\Phi(x, t)=\varphi(x) e^{\frac{i}{\hbar c} \Lambda(x)} e^{-\frac{i}{\hbar} E t}
$$


where $\varphi$ satisfies the time-independent $\mathrm{KG}$ equation

$$
-\frac{\hbar^{2}}{2 m} \varphi^{\prime \prime}+V_{\text {eff }} \varphi=E_{\text {eff }} \varphi
$$

with

$$
E_{\text {eff }}=\frac{E^{2}-\left(m c^{2}\right)^{2}}{2 m c^{2}}, \quad V_{\text {eff }}=\frac{V_{s}^{2}-V_{t}^{2}}{2 m c^{2}}+V_{s}+\frac{E}{m c^{2}} V_{t}
$$

and

$$
\Lambda(x)=\int^{x} d \eta V_{s p}(\eta)
$$

The density and flux corresponding to (41) are then

$$
\rho=\frac{E-V_{t}}{m c^{2}}|\varphi|^{2}, \quad J=\frac{\hbar}{2 i m}\left(\varphi^{*} \frac{\partial \varphi}{\partial x}-\frac{\partial \varphi^{*}}{\partial x} \varphi\right)
$$

Since $\rho$ and $J$ are independent of time, $\varphi$ is said to describe a stationary state. Notice that the density becomes negative in regions of space where $V_{t}>E$, so that the KG wave function must be normalized as

$$
\int_{-\infty}^{+\infty} d x \frac{E-V_{t}(x)}{m c^{2}}|\varphi(x)|^{2}= \pm 1
$$

where the \pm sign must be used for

$$
E \gtrless \frac{\int_{-\infty}^{+\infty} d x V_{t}(x)|\varphi(x)|^{2}}{\int_{-\infty}^{+\infty} d x|\varphi(x)|^{2}}
$$

Meanwhile, in the nonrelativistic approximation (potential energies small compared to $m c^{2}$ and $E \simeq m c^{2}$ ) Eq. (5) becomes

$$
\left(-\frac{\hbar^{2}}{2 m} \frac{d^{2}}{d x^{2}}+V_{t}+V_{s}\right) \varphi=\left(E-m c^{2}\right) \varphi
$$

so that $\varphi$ obeys the Schrödinger equation with binding energy equal to $E-m c^{2}$ without distinguishing the contributions of vector and scalar potentials. Furthermore, the density and current (and the normalization condition with the plus sign too) reduce precisely to the corresponding values of the nonrelativistic theory. 
It is remarkable that the KG equation with a scalar potential, or a vector potential contaminated with some scalar coupling, is not invariant under

$V \rightarrow V+$ const., this is so because the vector potential couples to the charge of the particle, whereas the scalar potential couples to the mass of the particle. Therefore, if there is any scalar coupling the absolute values of the energy will have physical significance and the freedom to choose a zero-energy will be lost. As we will see explicitly in this work, a constant added to the scalar potential is undoubtedly physically relevant. As a matter of fact, it can play a crucial role to ensure the existence of bound-state solutions even though the bound states are not present in the nonrelativistic limit of the theory. It is well known that a binding potential in the nonrelativistic approach is not binding in the relativistic approach when it is considered as a Lorentz vector. It is not immediately obvious that relativistic binding potentials may only result in scattering states in the nonrelativistic approach. The secret lies in the fact that vector and scalar potentials couple differently in the KG equation whereas there is no such distinction among them in the Schrödinger equation. This observation permit us to conclude that even a "repulsive" potential might be a bona fide binding potential.

\section{The mixed vector-scalar kink-like potential and the effective Rosen-Morse potential}

Now let us focus our attention on scalar and vector potentials in the form of smooth steps:

$$
\begin{aligned}
& V_{t}=g_{t} V, \quad V_{s}=g_{s}(\mathcal{V}+V) \\
& \mathcal{V}=\text { const }, \quad V=\frac{V_{0}}{2}\left(1+\tanh \frac{x}{2 L}\right)
\end{aligned}
$$

where the dimensionless coupling constants, $g_{t}$ and $g_{s}$, are real numbers constrained by $g_{t}+g_{s}=1$. The positive parameter $L$ is related to the range of the interaction which makes $V$ to change noticeably in the interval $-2 L<x<2 L$, and as $L \rightarrow 0$ the potential approximates the square step potential. $V_{0}>0$ is the height of the potential $V$ at $x=+\infty$. The uniform background makes the height of the scalar potential at $x=-\infty$ to be $g_{s} \mathcal{V}$. 
The reason for including a scalar uniform background will be clear later - it makes possible the existence of bound-state solutions.

Before proceeding, it is useful to note that the effective potential corresponding to (12) is recognized as the exactly solvable Rosen-Morse potential [16]-[17]

$$
V_{\text {eff }}=-V_{1} \operatorname{sech}^{2} \frac{x}{2 L}+V_{2} \tanh \frac{x}{2 L}+V_{3}
$$

where the following abbreviations have been used

$$
\begin{gathered}
V_{1}=\left(2 g_{s}-1\right) \frac{V_{0}^{2}}{8 m c^{2}} \\
V_{2}=2 V_{1}+\frac{V_{0}}{2 m c^{2}}\left(g_{s} M c^{2}+g_{t} E\right) \\
V_{3}=V_{2}+E_{\text {eff }}-\frac{E^{2}-\left(M c^{2}\right)^{2}}{2 m c^{2}}
\end{gathered}
$$

and

$$
M=m+g_{s} \frac{\mathcal{V}}{c^{2}}
$$

The Rosen-Morse potential approaches $V_{3} \pm V_{2}$ as $x \rightarrow \pm \infty$, and has an extremum when $\left|V_{2}\right|<2\left|V_{1}\right|$ at

$$
x_{m}=L \ln \left(\frac{2 V_{1}-V_{2}}{2 V_{1}+V_{2}}\right)
$$

given by

$$
V_{\text {eff }}\left(x_{m}\right)=V_{3}-V_{1}\left[1+\left(\frac{V_{2}}{2 V_{1}}\right)^{2}\right]
$$

The second derivative of $V_{\text {eff }}$ at $x_{m}$ is given by

$$
V_{\text {eff }}^{\prime \prime}\left(x_{m}\right)=\frac{1}{\left(2 V_{1}\right)^{3}}\left[\frac{\left(2 V_{1}\right)^{2}-V_{2}^{2}}{2 L}\right]^{2}
$$

in such a way that the extremum is a local minimum (maximum) only if $V_{1}>0\left(V_{1}<0\right)$. Note that the extremum, if it exists at all, is a minimum 
(maximum) only if $g_{s}>1 / 2\left(g_{s}<1 / 2\right)$. In particular, the symmetric RosenMorse potential is that one with $V_{2}=0$ which can be obtained with

$$
g_{s}^{2} \mathcal{V}=-\left[g_{s} m c^{2}+g_{t} E+\left(2 g_{s}-1\right) \frac{V_{0}}{2}\right]
$$

As a matter of fact, potential-well structures can be achieved when $\left|V_{2}\right|<$ $2\left|V_{1}\right|$ with $V_{1}>0$ and a pressing need for bound-state solutions implies that $E_{\text {eff }}$ defined in (6) must satisfy $V_{\text {eff }}\left(x_{m}\right)<E_{\text {eff }}<V_{\text {eff }}( \pm \infty)$. From the condition $E_{\text {eff }}<V_{\text {eff }}( \pm \infty)$ one concludes that

$$
|E|<|M| c^{2}, \quad\left|E-g_{t} V_{0}\right|<\left|M c^{2}+g_{s} V_{0}\right|
$$

Writing $V_{\text {eff }}\left(x_{m}\right)$ as

$$
V_{\text {eff }}\left(x_{m}\right)=V_{2}-V_{1}\left[1+\left(\frac{V_{2}}{2 V_{1}}\right)^{2}\right]+E_{\text {eff }}-\frac{E^{2}-\left(M c^{2}\right)^{2}}{2 m c^{2}}
$$

one can see that the condition $E_{\text {eff }}>V_{\text {eff }}\left(x_{m}\right)$ turns into

$$
E \neq-\frac{g_{t}}{g_{s}} M c^{2}
$$

According to this last result, there is no crossing between energy levels with $E>-g_{t} M c^{2} / g_{s}$ (to be associated with the particle levels) and those ones with $E<-g_{t} M c^{2} / g_{s}$ (to be associated with the particle levels). This fact implies that there is no channel for spontaneous particle-antiparticle creation, so that the single-particle interpretation of the KG equation is preserved. In particular, null energies are not permissible for bound-state solutions in the case of a pure scalar coupling $\left(g_{s}=1\right)$, when the energy levels are disposed symmetrically about $E=0$. Furthermore, the coefficient $V_{2}$ in (13) can also be expressed as

$$
V_{2}=-2 V_{1}+\frac{V_{0}}{2 m c^{2}}\left[g_{s} M c^{2}+g_{t} E+\left(2 g_{s}-1\right) V_{0}\right]
$$

From this and from the definition of $V_{2}$ in (14), it follows that

$$
g_{s} M c^{2}+g_{t} E<0
$$

and

$$
g_{s} M c^{2}+g_{t} E+\left(2 g_{s}-1\right) V_{0}>0
$$


Combining these two inequalities one concludes that an additional sine qua non condition for the existence of bounded solutions is that $M c^{2}$ must be into the limits

$$
-\left(\frac{2 g_{s}-1}{g_{s}} V_{0}+\frac{g_{t}}{g_{s}} E\right)<M c^{2}<-\frac{g_{t}}{g_{s}} E
$$

To acknowledge that the effective potential for the mixing given by (12) is a Rosen-Morse potential can help you to see more clearly how a kinklike smooth step potential might furnish bound-state solutions. After all, we shall not use the knowledge about the exact analytical solution for the Rosen-Morse potential.

\subsection{The asymptotic solutions}

As $|x| \gg L$ the effective potential is practically constant (the main transition region occurs in $|x|<2 L$ ) and the solutions for the KG equation can be approximate by those ones for a free particle. Furthermore, the asymptotic behaviour will show itself suitable to impose the appropriate boundary conditions to the complete solution to the problem.

The vector and scalar potentials approaches to zero and to $g_{s} \mathcal{V}$ as $x \rightarrow$ $-\infty$. Hence the solution for the KG equation can be written as

$$
\varphi(-\infty)=A_{+} e^{+i k x}+A_{-} e^{-i k x}
$$

where

$$
\hbar c k=\sqrt{E^{2}-\left(M c^{2}\right)^{2}}
$$

For $|E|>|M| c^{2}$, the solution expressed by (27) describes plane waves propagating on both directions of the $X$-axis with group velocity $v_{g}=(d E / d k) / \hbar$ equal to the classical velocity. If we consider that particles are incident on the potential $\left(E>|M| c^{2}\right), A_{+} \exp (+i k x)$ will describe particles coming to the potential region from $-\infty\left(v_{g}=+c^{2} \hbar k / E>0\right)$, whereas $A_{-} \exp (-i k x)$

will describe reflected particles $\left(v_{g}=-c^{2} \hbar k / E<0\right)$. The flux corresponding to $\varphi$ given by (27), is expressed as

$$
J(-\infty)=J_{\text {inc }}-J_{\text {ref }}
$$

where 


$$
J_{\text {inc }}=\frac{\hbar k}{m}\left|A_{+}\right|^{2}, \quad J_{\text {ref }}=\frac{\hbar k}{m}\left|A_{-}\right|^{2}
$$

Note that the relation $J=\rho v_{g}$ maintains for the incident and reflected waves, since

$$
\rho_{ \pm}(-\infty)=\frac{E}{m c^{2}}\left|A_{ \pm}\right|^{2}
$$

On the other hand, the vector and scalar potentials approaches to $V_{0}$ and to $g_{s}\left(\mathcal{V}+V_{0}\right)$ as $x \rightarrow+\infty$. In this asymptotic region one should have $v_{g} \geq 0$ in such a way that the solution in this region of space describes an evanescent wave or a progressive wave running away from the potential potential region. The general solution has the form

$$
\varphi(+\infty)=B_{+} e^{+i \kappa x}+B_{-} e^{-i \kappa x}
$$

where

$$
\hbar c \kappa=\sqrt{\left(E-g_{t} V_{0}\right)^{2}-\left(M c^{2}+g_{s} V_{0}\right)^{2}}
$$

Due to the twofold possibility of signs for the energy of a stationary state, the solution involving $B_{-}$can not be ruled out a priori. As a matter of fact, this term may describe a progressive wave with negative charge density and phase velocity $v_{p h}=|E| /(\hbar \kappa)>0$. It is true that if $\kappa \in \mathbb{R}$ the solution describing a plane wave propagating in the positive direction of the $X$-axis with group velocity $v_{g}= \pm c^{2} \hbar \kappa /\left(E-g_{t} V_{0}\right)>0$ is possible only if $E \gtrless g_{t} V_{0}$ with $B_{\mp}=0$. In this case the density and the flux corresponding to $\varphi$ given by (32) are expressed as

$$
\rho(+\infty)=\frac{E-g_{t} V_{0}}{m c^{2}}\left|B_{ \pm}\right|^{2}, \quad J(+\infty)=J_{\text {trans }}= \pm \frac{\hbar \kappa}{m}\left|B_{ \pm}\right|^{2}
$$

If $\kappa$ is imaginary one can write $\kappa= \pm i Q$ with $Q \in \mathbb{R}$, and (32) with $B_{\mp}=0$ describes an evanescent wave $\left(v_{g}=0\right)$. The condition $B_{\mp}=0$ is necessary for furnishing a finite charge density as $x \rightarrow \infty$. In this case

$$
\rho(+\infty)=\frac{E-g_{t} V_{0}}{m c^{2}}\left|B_{ \pm}\right|^{2} e^{-2 Q x}, \quad J(+\infty)=0
$$

When $\kappa \in \mathbb{R}$, a bizarre circumstance occurs as long as $E<g_{t} V_{0}$ since both $\rho(+\infty)$ and $J(+\infty)$ are negative quantities. The maintenance of the relation 
$J=\rho v_{g}$, though, is a license to interpret the solution $B_{-} e^{-i \kappa x}$ as describing the propagation, in the positive direction of the $X$-axis, of particles with charges of opposite sign to the incident particles. This interpretation is consistent if the particles moving in this asymptotic region have energy $-E$ and are under the influence of a potential $-g_{t} V_{0}$. It means that, in fact, the progressive wave describes the propagation of antiparticles in the positive direction of the $X$-axis. If $\kappa$ is imaginary, though, the solution with $E>g_{t} V_{0}\left(E<g_{t} V_{0}\right)$ describes an evanescent wave associated with particles (antiparticles).

Defining

$$
\varepsilon=\frac{g_{t}}{1-g_{t}}|M| c^{2}, \quad V_{c}=\left\{\begin{array}{cl}
\frac{E+M c^{2}}{2 g_{t}-1}, & \text { for } \quad g_{t}>\frac{1}{2} \\
\infty, & \text { for } \quad g_{t} \leq \frac{1}{2}
\end{array}\right.
$$

and the following cases

- Case I. $M>0$

- Case II. $M<0$ with $g_{t} \leq 1 / 2$

- Case III. $M<0$ with $1 / 2<g_{t}<1$ and $E>\varepsilon$

- Case IV. $M<0$ with $1 / 2<g_{t}<1$ and $E<\varepsilon$

one can readily envisage that the segregation between $\kappa$ real and $\kappa$ imaginary allows us to identify three distinct class of scattering solutions for particles depending on $V_{0}$ :

- Class A. $\kappa$ is real with $V_{0}<E-M c^{2}$ for the cases I, II, III, and $V_{0}<V_{c}$ for the case IV.

- Class B. $\kappa$ is imaginary with $E-M c^{2}<V_{0}<V_{c}$ for the cases I, II, III, and $V_{c}<V_{0}<E-M c^{2}$ for the case IV.

- Class C. $\kappa$ is real with $V_{0}>V_{c}$ for the cases I, III, and $V_{0}>E-M c^{2}$ for the case IV.

Now we focus attention on the calculation of the reflection $(R)$ and transmission $(T)$ coefficients. The reflection (transmission) coefficient is defined 
as the ratio of the reflected (transmitted) flux to the incident flux. Since $\partial \rho / \partial t=0$ for stationary states, one has that $J$ is independent of $x$. This fact implies that

$$
T=\left\{\begin{array}{cc} 
\pm \frac{\kappa}{k} \frac{\left|B_{ \pm}\right|^{2}}{\left|A_{+}\right|^{2}}, & \text { for } \kappa \in \mathbb{R},\left.B_{\mp}\right|^{2} \\
0, & \text { for } \kappa= \pm i Q
\end{array}\right.
$$

For all the cases one should have $R+T=1$, as expected for a conserved quantity. This fact is easily verified for $\kappa$ imaginary. For $\kappa$ real one has to wait for the complete solution of the problem whose asymptotic behaviour allows one to calculate the amplitudes of all waves relative to amplitude of the incident wave. Is is instructive to note that the case with $B_{+}=0$ presents $R>1$, the alluded Klein's paradox, implying that more particles are reflected from the potential region than those incoming. Note that for $\mathcal{V}>-m c^{2} / g_{s}(M>0)$ the threshold for pair production is equal to $2 m c^{2}$ for $g_{t}=1$ and greater than $2 m c^{2}$ for $1 / 2<g_{t}<1$. For $\mathcal{V}<-m c^{2} / g_{s}$ $\left(M<0, g_{t}<1\right)$, though, one has that the threshold is equal to $2|M| c^{2}$, and that the threshold tends to zero as $\mathcal{V}$ tends to $-m c^{2} / g_{s}$. In this last circumstance, pair production occurs for every $V_{0}$, however small.

It is worthwhile to note that the asymptotic solutions with $k= \pm i q$, where $q \in \mathbb{R}$, and $\kappa= \pm i Q$, might describe the possible existence of bound states realized beforehand in the previous section, since $J( \pm \infty)=0$. In this case, one has to impose that $A_{ \pm}=0$ for $k= \pm i q$.

\subsection{The complete solutions}

Armed with the knowledge about asymptotic solutions and with the definitions of the reflection and transmission coefficients we proceed for searching solutions on the entire region of space.

Changing the independent variable $x$ in (5) to

$$
y=\frac{1}{2}\left(1-\tanh \frac{x}{2 L}\right)
$$

the $\mathrm{KG}$ equation is transformed into the differential equation for $\varphi(y)$ : 


$$
y(1-y) \varphi^{\prime \prime}+(1-2 y) \varphi^{\prime}+\Theta \varphi=0
$$

where

$$
\Theta=\left(\frac{L}{\hbar c}\right)^{2} \frac{\left[E-g_{t} V_{0}(1-y)\right]^{2}-\left\{m c^{2}+g_{s}\left[\mathcal{V}+V_{0}(1-y)\right]\right\}^{2}}{y(1-y)}
$$

Introducing a new function $\psi(y)$ through the relation

$$
\varphi(y)=y^{\nu}(1-y)^{\mu} \psi(y)
$$

and defining

$$
\begin{gathered}
a=\mu+\nu+\frac{1-\omega}{2}, \quad b=\mu+\nu+\frac{1+\omega}{2}, \quad C=2 \nu+1 \\
\mu^{2}=-(k L)^{2}, \quad \nu^{2}=-(\kappa L)^{2}, \quad \omega^{2}=1+\left(2 g_{s}-1\right)\left(\frac{2 L V_{0}}{\hbar c}\right)^{2}
\end{gathered}
$$

the equation (40) becomes the hypergeometric differential equation [18]

$$
y(1-y) \psi^{\prime \prime}+[C-(a+b+1) y] \psi^{\prime}-a b \psi=0
$$

whose general solution can be written in terms of the Gauss hypergeometric series

$$
{ }_{2} F_{1}(a, b, C, y)=\frac{\Gamma(C)}{\Gamma(a) \Gamma(b)} \sum_{n=0}^{\infty} \frac{\Gamma(a+n) \Gamma(b+n)}{\Gamma(C+n)} \frac{y^{n}}{n !}
$$

in the form [18]

$$
\psi=A_{2} F_{1}(a, b, C, y)+B y_{2}^{-2 \nu} F_{1}(a+1-C, b+1-C, 2-C, y)
$$

in such a way that

$$
\varphi=A y^{\nu}(1-y)_{2}^{\mu} F_{1}(a, b, C, y)
$$




$$
+B y^{-\nu}(1-y)_{2}^{\mu} F_{1}(a+1-C, b+1-C, 2-C, y)
$$

with the constants $A$ and $B$ to be fitted by the asymptotic behaviour analyzed in the previous discussion.

As $x \rightarrow+\infty$ (that is, as $y \rightarrow 0)$, one has that $y \simeq \exp (-x / L)$ and (47), because ${ }_{2} F_{1}(a, b, C, 0)=1$, reduces to

$$
\varphi(+\infty) \simeq A e^{-\nu x / L}+B e^{\nu x / L}
$$

so the asymptotic behaviour requires that $B=0$, and $A=B_{ \pm}$for $\nu=\mp i \kappa L$. The asymptotic behaviour as $x \rightarrow-\infty(y \rightarrow 1)$ can be found by using the relation for passing over from $y$ to $1-y$ :

$$
\begin{gathered}
{ }_{2} F_{1}(a, b, C, y)=\gamma_{-2} F_{1}(a, b, a+b-C+1,1-y) \\
+\gamma_{+}{ }_{2} F_{1}(C-a, C-b, C-a-b+1,1-y)(1-y)^{C-a-b}
\end{gathered}
$$

where $\gamma_{+}$and $\gamma_{-}$are expressed in terms of the gamma function as

$$
\gamma_{-}=\frac{\Gamma(C) \Gamma(C-a-b)}{\Gamma(C-a) \Gamma(C-b)}, \quad \gamma_{+}=\frac{\Gamma(C) \Gamma(a+b-C)}{\Gamma(a) \Gamma(b)}
$$

which can also be written as

$$
\begin{aligned}
& \gamma_{-}=\frac{\Gamma(2 \nu+1) \Gamma(-2 \mu)}{\Gamma\left(\frac{1+\omega}{2}+\nu-\mu\right) \Gamma\left(\frac{1-\omega}{2}+\nu-\mu\right)} \\
& \gamma_{+}=\frac{\Gamma(2 \nu+1) \Gamma(2 \mu)}{\Gamma\left(\frac{1+\omega}{2}+\nu+\mu\right) \Gamma\left(\frac{1-\omega}{2}+\nu+\mu\right)}
\end{aligned}
$$

Now, as $x \rightarrow-\infty, 1-y \simeq \exp (+x / L)$. This time, (47) tends to

$$
\varphi(-\infty) \simeq A \gamma_{+} e^{-\mu x / L}+A \gamma_{-} e^{+\mu x / L}
$$

so that $A \gamma_{ \pm}=A_{ \pm}$for $\mu=-i k L$, in accordance with the previous analysis for very large negative values of $x$.

Therefore, the asymptotic behaviour of the general solution dictates that $B=0$ and establishes conditions on $\mu$ and $\nu$, but not on $\omega$. The reflection (37) and transmission (38) coefficients can now be expressed as 


$$
\begin{gathered}
R=\frac{\left|\gamma_{-}\right|^{2}}{\left|\gamma_{+}\right|^{2}}=\frac{\left|\Gamma\left(\frac{1+\omega}{2}+\nu+\mu\right) \Gamma\left(\frac{1-\omega}{2}+\nu+\mu\right)\right|^{2}}{\left|\Gamma\left(\frac{1+\omega}{2}+\nu-\mu\right) \Gamma\left(\frac{1-\omega}{2}+\nu-\mu\right)\right|^{2}} \frac{|\Gamma(-2 \mu)|^{2}}{|\Gamma(2 \mu)|^{2}} \\
T=\left\{\begin{array}{cc}
\frac{\nu}{\mu} \frac{1}{\left|\gamma_{+}\right|^{2}}=\frac{\nu}{\mu} \frac{\left|\Gamma\left(\frac{1+\omega}{2}+\nu+\mu\right) \Gamma\left(\frac{1-\omega}{2}+\nu+\mu\right)\right|^{2}}{|\Gamma(2 \nu+1) \Gamma(2 \mu)|^{2}}, & \text { for } \quad \nu=\mp i \kappa L \\
0, & \text { for } \quad \nu \in \mathbb{R}
\end{array}\right.
\end{gathered}
$$

In the numerical evaluation of $R$ and $T$ one has not only to distinguish the sign of the imaginary part of $\nu$ but also if or not $\omega$ is real. The following identities involving the gamma function [18]

$$
\Gamma\left(z^{*}\right)=\Gamma^{*}(z), \quad \Gamma(z) \Gamma(1-z)=\frac{\pi}{\sin (\pi z)}
$$

are sufficient enough to show that

$$
|\Gamma(u+i v) \Gamma(1-u+i v)|^{2}=\frac{2 \pi^{2}}{\cosh (2 \pi v)-\cos (2 \pi u)}
$$

where $u$ and $v$ are the real and imaginary parts of $z$. Furthermore, the following identities will be useful [18]

$$
|\Gamma(i v)|^{2}=\frac{\pi}{v \sinh (\pi v)}, \quad|\Gamma(1+i v)|^{2}=\frac{\pi v}{\sinh (\pi v)}
$$

Hence, one can find for $\mu=-i k L$ and $\nu=\mp i \kappa L$ :

$$
\begin{gathered}
R=\left\{\begin{array}{cc}
\frac{\cosh [2 \pi(k \mp \kappa) L]-\cos [\pi(1+\omega)]}{\cosh [2 \pi(k \pm \kappa) L]-\cos [\pi(1+\omega)]} \lessgtr 1, & \text { for } \omega \in \mathbb{R} \\
\frac{\cosh [\pi(k \mp \kappa+N) L] \cosh [\pi(k \mp \kappa-N) L]}{\cosh [\pi(k \pm \kappa-N) L] \cosh [\pi(k \pm \kappa+N) L]} \lessgtr 1, & \text { for } \quad \omega=2 i N L, N \in \mathbb{R}
\end{array}\right. \\
T=\left\{\begin{array}{cc} 
\pm \frac{2 \sinh (2 \pi k L) \sinh (2 \pi \kappa L)}{\cosh [2 \pi(k \pm \kappa) L]-\cos [\pi(1+\omega)]} \gtrless 0, & \text { for } \omega \in \mathbb{R} \\
\pm \frac{\sinh (2 \pi k L) \sinh (2 \pi \kappa L)}{\cosh [\pi(k \pm \kappa-N) L] \cosh [\pi(k \pm \kappa+N) L]} \gtrless 0, & \text { for } \quad \omega=2 i N L, N \in \mathbb{R}
\end{array}\right.
\end{gathered}
$$

whereas for $\mu=-i k L$ and $\nu \in \mathbb{R}$ ( $\kappa$ pure imaginary) one has $T=0$ and 


$$
R=\frac{\left|\Gamma\left(\frac{1+\omega}{2}+\nu-i k L\right) \Gamma\left(\frac{1-\omega}{2}+\nu-i k L\right)\right|^{2}}{\left|\Gamma\left(\frac{1+\omega}{2}+\nu+i k L\right) \Gamma\left(\frac{1-\omega}{2}+\nu+i k L\right)\right|^{2}}=1, \forall \omega
$$

At any circumstance, from the hyperbolic trigonometric identities involving $\cosh \left(z_{1}+z_{2}\right)$, one can easily show that $R+T=1$ and that as $\omega \rightarrow 1$ one finds:

$$
\begin{gathered}
R=\left\{\begin{array}{cc}
\left(\frac{k \mp \kappa}{k \pm \kappa}\right)^{2}, & \text { for } \mu=-i k L, \nu=\mp i \kappa L \\
1, & \text { for } \mu=-i k L, \nu \in \mathbb{R}
\end{array}\right. \\
T=\left\{\begin{array}{cc} 
\pm \frac{4 k \kappa}{(k \pm \kappa)^{2}}, & \text { for } \mu=-i k L, \nu=\mp i \kappa L \\
0, & \text { for } \mu=-i k L, \nu \in \mathbb{R}
\end{array}\right.
\end{gathered}
$$

Note that (61) and (62) reduce to the results for the square step potential [2], 4], as they should be since $\omega \rightarrow 1$ as $L \rightarrow 0$. Now, $R$ and $T$ blow up for $\kappa=k$ (Class C), i.e. when (19) is satisfied. Of course, this crisis never mentioned in the literature does not mean that the KG theory fails. It only means that the calculations lose their validity for discontinuous potentials.

\subsection{Bound states}

As we have said, the solution expressed by (47) with $\mu$ and $\nu$ as real quantities ( $k$ and $\kappa$ as imaginary numbers), viz.

$$
\begin{aligned}
& \mu=\frac{L}{\hbar c} \sqrt{\left(M c^{2}\right)^{2}-E^{2}} \\
& \nu=\frac{L}{\hbar c} \sqrt{\left(M c^{2}+g_{s} V_{0}\right)^{2}-\left(E-g_{t} V_{0}\right)^{2}}
\end{aligned}
$$

might describe the possible existence of bound states by imposing that $B=0$ and $\gamma_{+}=0$. In view of (50) one has to locate the singular points of $\Gamma(a) \Gamma(b)$. It happens that $\Gamma(z)$ has simple poles only on the real axis at $z=-n$ $(n=0,1,2, \ldots)$, and invoking the expression of $a$ and $b$ in terms of $\mu, \nu$ and $\omega$ given by (43) one concludes that $\omega$ has got to be a real number. Evidently $L V_{0}$ must be chosen such that 


$$
L V_{0}<\left\{\begin{array}{cl}
\infty, & \text { for } g_{s} \geqslant 1 / 2 \\
\frac{\hbar c}{2 \sqrt{1-2 g_{s}}}, & \text { for } g_{s}<1 / 2
\end{array}\right.
$$

The quantization condition is thus given by $a=-n$ for $\omega>0$ and $b=-n$ for $\omega<0$. Since ${ }_{2} F_{1}(a, b, C, y)$ is invariant under exchange of $a$ and $b$, one obtains a quantization condition independent of the sign of $\omega$ :

$$
\mu+\nu+\frac{1-|\omega|}{2}=-n, n=0,1,2, \ldots
$$

Eq. (65) can also be written in the form

$$
\begin{aligned}
& \sqrt{\left(M c^{2}\right)^{2}-E^{2}}+\sqrt{\left(M c^{2}+g_{s} V_{0}\right)^{2}-\left(E-g_{t} V_{0}\right)^{2}} \\
= & \frac{\hbar c}{2 L}\left[\sqrt{1+\left(2 g_{s}-1\right)\left(\frac{2 L V_{0}}{\hbar c}\right)^{2}}-(2 n+1)\right], n=0,1,2, \ldots
\end{aligned}
$$

Now we have an irrational algebraic equation to be solved numerically, but there are still some questions that one ought to get answered. Does it furnish proper solutions for the KG equation? Evidently $\varphi$ as in (47) is a squareintegrable function, and $\mu$ and $\nu$ must be positive in order to furnish a wave function vanishing at $x= \pm \infty$. Hence, the following supplementary conditions must be imposed:

$$
|E|<|M| c^{2}, \quad\left|E-g_{t} V_{0}\right|<\left|M c^{2}+g_{s} V_{0}\right|
$$

as given by (20), and

$$
g_{s}>1 / 2, \quad n=0,1,2, \ldots<s
$$

where

$$
s=\frac{1}{2}\left[-1+\sqrt{1+\left(2 g_{s}-1\right)\left(\frac{2 L V_{0}}{\hbar c}\right)^{2}}\right]
$$

The first pair of supplementary conditions ensures that $\mu$ and $\nu$ are positive. The second pair is necessary to make positive the right-hand side of (66). 
Note that this last pair of supplementary conditions imposes an additional restriction on the product $L V_{0}$ beyond that one which makes $\omega$ a real number as given by (64). As a matter of fact, those conditions kill all the possibilities for bound states if the scalar coupling does not exceeds the vector coupling. At any rate, the possible solutions of (66) constitute a finite set of solutions. According to the second line of (68) and (69) one has

$$
L V_{0}>\hbar c \sqrt{\frac{n(n+1)}{2 g_{s}-1}}
$$

This means that the number of allowed bound states increase with $L V_{0}$, and there is at least one solution, no matter how small is $L V_{0}$. Now the Gauss hypergeometric series ${ }_{2} F_{1}(a, b, C, y)$ reduces to nothing but a polynomial of degree $n$ in $y$ when $a$ or $b$ is equal to $-n$ : Jacobi's polynomial of index $\alpha$ and $\beta$. Indeed, for $a=-n$ one has [18]

$$
\begin{aligned}
{ }_{2} F_{1}(a, b, C, y) & ={ }_{2} F_{1}(-n, \alpha+1+\beta+n, \alpha+1, y) \\
& =\frac{n !}{(\alpha+1)_{n}} P_{n}^{(\alpha, \beta)}(\xi)
\end{aligned}
$$

where

$$
\alpha=2 \nu, \quad \beta=2 \mu, \quad \xi=1-2 y=\tanh \frac{x}{2 L}
$$

and $(\alpha)_{n}=\alpha(\alpha+1)(\alpha+2) \ldots(\alpha+n-1)$ with $(\alpha)_{0}=1$. Jacobi's polynomials $P_{n}^{(\alpha, \beta)}(\xi)$ are orthogonal with respect to the weighting function $w^{(\alpha, \beta)}(\xi)=(1-\xi)^{\alpha}(1+\xi)^{\beta}$ on the interval $[-1,+1]$, and can be standardized as

$$
P_{n}^{(\alpha, \beta)}(1)=\frac{(\alpha+1)_{n}}{n !}
$$

so that

$$
\int_{-1}^{+1} d \xi w^{(\alpha, \beta)}(\xi) P_{n}^{(\alpha, \beta)}(\xi) P_{n^{\prime}}^{(\alpha, \beta)}(\xi)=\delta_{n n^{\prime}} h_{n}^{(\alpha, \beta)}
$$

where $h_{n}^{(\alpha, \beta)}$ is given by [18] 


$$
h_{n}^{(\alpha, \beta)}=\frac{2^{\alpha+\beta+1}}{2 n+\alpha+\beta+1} \frac{\Gamma(n+\alpha+1) \Gamma(n+\beta+1)}{n ! \Gamma(n+\alpha+\beta+1)}
$$

Hence the KG wave function can be written as (see Ref. [17]):

$$
\varphi_{n}(\xi)=N_{n}(1-\xi)^{\alpha / 2}(1+\xi)^{\beta / 2} P_{n}^{(\alpha, \beta)}(\xi)
$$

where $N_{n}$ is given by

$$
N_{n}=\sqrt{\frac{\alpha \beta}{2^{\alpha+\beta} L(\alpha+\beta)} \frac{\Gamma(\alpha+\beta+n+1) \Gamma(n+1)}{\Gamma(\alpha+n+1) \Gamma(\beta+n+1)}}
$$

in such a manner that

$$
\int_{-\infty}^{+\infty} d x\left|\varphi_{n}(x)\right|^{2}=1
$$

and

$$
\int_{-\infty}^{+\infty} d x V_{t}(x)\left|\varphi_{n}(x)\right|^{2}=V_{0} \frac{\beta}{\beta+\alpha}
$$

Nevertheless, using (9) and (10) one can show that the normalized KG wave function must be written as

$$
\varphi_{n}^{(N)}(\xi)=N_{ \pm} \varphi_{n}(\xi)
$$

with

$$
N_{ \pm}=\sqrt{ \pm m c^{2} \frac{\alpha+\beta}{(\alpha+\beta) E-g_{t} V_{0} \beta}}, \quad \pm \text { for } \quad E \gtrless g_{t} V_{0} \frac{\beta}{\beta+\alpha}
$$

It is worthwhile to note that invoking the property $P_{n}^{(\alpha, \beta)}(-\xi)=(-1)^{n} P_{n}^{(\beta, \alpha)}(\xi)$, one can conclude that the KG wave functions with definite parities (putting $\alpha=\beta$ ), associated with an even potential, are obtained on the condition that the uniform background satisfies (19), i.e. the effective potential is the symmetric Rosen-Morse potential. 


\section{Conclusions}

We have explored the influence of a scalar uniform background added to a mixed vector-scalar smooth step potential. We have verified that the background presents drastic effects on both scattering and bound-state solutions. The background increases the threshold for the pair production when the vector coupling exceeds the scalar coupling and $\mathcal{V}>-m c^{2} / g_{s}$. On the other hand, if $\mathcal{V}<-m c^{2} / g_{s}$ the background decreases the threshold and pair production may occur for a potential barrier arbitrarily small. When the scalar coupling exceeds the vector coupling there appears the possibility of a finite set of bound-state solutions. It is curious that the smooth step potential might hold bound states in spite of the fact that the potential given by (12) is everywhere repulsive, so that one can not expect bound states in the nonrelativistic limit. Of course, the scalar uniform background plays a peremptory role for the actual occurrence of bound states but no nonrelativistic limit can expected since the background has to be in a range of values which do not acquiesce a nonrelativistic limit.

\section{Acknowledgments}

This work was supported in part through funds provided by CNPq. 


\section{References}

[1] W. Greiner, Relativistic Quantum Mechanics, Wave Equations, Springer-Verlag, Berlin, 1990;

P. Strange, Relativistic Quantum Mechanics with Applications in Condensed Matter and Atomic Physics, Cambridge University Press, Cambridge 1998;

J.D. Bjorken, S.D. Drell, Relativistic Quantum Mechanics, McGrawHill, New York, 1964;

J.J. Sakurai, Advanced Quantum Mechanics, Addison-Wesley, Reading 1967.

[2] F. Gross, Relativistic Quantum Mechanics and Field Theory, Wiley, New York, 1993.

[3] O. Klein, Z. Phys. 53 (1929) 157.

[4] R.G. Winter, Am. J. Phys. 27 (1959) 355;

M.G. Fu, E. Furlani, Am. J. Phys. 50 (1982) 545;

B.R. Holstein, A.m J. Phys. 66 (1998) 507;

J.-J. Ni, W. Zhou, J. Yan, Klein Paradox and Antiparticle, arXiv: quant-ph/9905044;

J. Villavicencio, J. Phys. A 33 (2000) 6061;

T.R. Cardoso, A.S. de Castro, Rev. Bras. Ens. Fís. 29 (2007) 203.

[5] See, e.g., R. Rajaraman, Solitons and Instantons, New-Holland, Amsterdan, 1982.

[6] J. Goldstone, F. Wilczek, Phys. Rev. Lett. 47 (1981) 986;

R. Jackiw, G. Semenoff, Phys. Rev. Lett. 50 (1983) 439;

For a review see: A.J. Niemi, G. Semenoff, Phys. Rep. 135 (1986) 99.

[7] A.S. de Castro, M. Hott, Phys. Lett. A 351 (2006) 379.

[8] S. Jeschonnek, J.W. Van Orden, Phys. Rev. D 69 (2004) 054006. 
[9] Y. Salamin, et al., Phys. Rep. 427 (2006)41;

M. Klaiber, Phys. Rev. A 75 (2007) 063413.

[10] R.N. Lee, A.I. Milstein, S.G. Karshenboim, Phys. Rev. A 73 (2006) 012505.

[11] G. Parzen, Phys. Rev. 81 (1951) 808;

L.I. Schiff, Phys. Rev. 103 (1956) 443;

G. Parzen, Phys. Rev. 104 (1956) 835.

[12] W. Lucha, et al., Phys. Rep. 200 (1991) 127 and references therein.

[13] B.D. Serot, J.D. Walecka, in: Advances in Nuclear Physics, Vol. 16, edited by J.W. Negele and E. Vogt, Plenum, New York, 1986;

J.N. Ginocchio, Phys. Rev. Lett. 78 (1997) 436;

J.N. Ginocchio, A. Leviatan, Phys. Lett. B 425 (1998) 1;

J.N. Ginocchio, Phys. Rep. 315 (1999) 231;

P. Alberto, et al., Phys. Rev. Lett. 86 (2001) 5015;

P. Alberto, et al., Phys. Rev. C 65 (2002) 034307;

T.-S. Chen, et al., Chin. Phys. Lett. 20 (2003) 358;

G. Mao, Phys. Rev. C 67 (2003) 044318;

R. Lisboa, et al., Phys. Rev. C 69 (2004) 024319.

[14] P. Alberto, A.S. de Castro, M. Malheiro, Phys. Rev. C 75 (2007) 047303.

[15] See, e.g., O.M. Braun, Y.S. Kivshar, The Frenkel-Kontorova Model: Concepts, Methods, and Applications, Springer, Berlin, 2004.

[16] N. Rosen, P.M. Morse, Phys. Rev. 42 (1932) 210;

G. Stanciu, Phys. Lett. 23 (1966) 232;

G. Stanciu, J. Math. Phys. 8 (1967) 2043.

[17] M.M. Nieto, Phys. Rev. A 17 (1978) 1273.

[18] M. Abramowitz, I.A. Stegun, Handbook of Mathematical Functions, Dover, Toronto, 1965. 